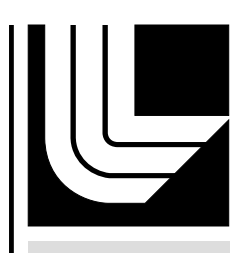

LA W RENCE LIVERM ORE NATIONAL LABORATORY

\title{
Convergent Ablator Performance Measurements
}

D. G. Hicks, B. K. Spears, D. G. Braun, R. E. Olson, C. M. Sorce, P. M. Celliers, G. W. Collins, O. L. Landen

July 16,2010

Physics of Plasmas 
This document was prepared as an account of work sponsored by an agency of the United States government. Neither the United States government nor Lawrence Livermore National Security, LLC, nor any of their employees makes any warranty, expressed or implied, or assumes any legal liability or responsibility for the accuracy, completeness, or usefulness of any information, apparatus, product, or process disclosed, or represents that its use would not infringe privately owned rights. Reference herein to any specific commercial product, process, or service by trade name, trademark, manufacturer, or otherwise does not necessarily constitute or imply its endorsement, recommendation, or favoring by the United States government or Lawrence Livermore National Security, LLC. The views and opinions of authors expressed herein do not necessarily state or reflect those of the United States government or Lawrence Livermore National Security, LLC, and shall not be used for advertising or product endorsement purposes. 


\title{
Convergent ablator performance measurements
}

\author{
D. G. Hicks, ${ }^{1, a)}$ B. K. Spears, ${ }^{1}$ D. G. Braun, ${ }^{1}$ R. E. Olson,${ }^{2}$ C. M. Sorce,${ }^{1}$ P. M. Celliers, ${ }^{1}$ G. W. Collins, ${ }^{1}$ and O. \\ L. Landen ${ }^{1}$ \\ 1) Lawrence Livermore National Laboratory, Livermore, CA 94550 \\ ${ }^{2)}$ Sandia National Laboratory, Albuquerque, New Mexico 87185
}

(Dated: 5 August 2010)

The velocity and remaining ablator mass of an imploding capsule are critical metrics for assessing the progress towards ignition of an inertially confined fusion experiment. These and other convergent ablator performance parameters have been measured using a single streaked x-ray radiograph. Traditional Abel inversion of such a radiograph is ill-posed since backlighter intensity profiles and x-ray attenuation by the ablated plasma are unknown. To address this we have developed a regularization technique which allows the ablator density profile, $\rho(r)$ and effective backlighter profile, $I_{0}(y)$, at each time step to be uniquely determined subject to the constraints that $\rho(r)$ is localized in radius space and $I_{0}(y)$ is delocalized in object space. Moments of $\rho(r)$ then provide the time-resolved areal density, mass, and average radius (and thus velocity) of the remaining ablator material. These results are combined in the spherical rocket model to determine the ablation pressure and mass ablation rate during the implosion. The technique has been validated on simulated radiographs of implosions at the National Ignition Facility [G. H. Miller et al., Nucl. Fusion 44, 228 (2004)] and implemented on experiments at the OMEGA laser facility [T. R. Boehly et al., Opt. Comm., 133, 495 (1997)].

\section{INTRODUCTION}

Inertial confinement fusion ${ }^{1}$ (ICF) aims to achieve thermonuclear ignition by compressing and heating matter inside a spherically-converging rocket. At the National Ignition Facility ${ }^{2}$ (NIF) the experimental approach to ignition involves measuring and tuning several key metrics each of which characterize different aspects of the implosion. ${ }^{3}$ These metrics include the laser-to-x-ray conversion efficiency, implosion symmetry, fuel adiabat, and fuel velocity. High fuel velocities are required to deliver compressive work to the hot spot faster than it is lost via conduction or radiation and is one of the most important figures-of-merit in ICF. Calculations show that the ignition threshold scales as the inverse sixth power of the velocity. ${ }^{4-6}$

Achieving a high fuel velocity involves a balance between two competing requirements: ${ }^{7}$ (1) Burning off most of the ablator mass to maximize the fuel kinetic energy, and (2) Preserving sufficient ablator mass to keep ablator-fuel interface instabilities and fuel pre-heat effects low. The maximum velocity desired is that which can be achieved with sufficiently low mix and pre-heat of the fuel. For typical indirectly-driven NIF ignition targets, radiation-hydrodynamic simulations have established that the optimal peak velocity is in the range 350$380 \mu \mathrm{m} / \mathrm{ns}$ while the remaining mass of the ablator is $5-15 \%$ of the initial ablator mass. The precise values depend sensitively upon the mix and transport models used in the simulations as well as on specifics of the target and laser drive.

For a given laser drive and target configuration, whether or not the desired velocity and mass are achieved

\footnotetext{
a) Author to whom correspondence should be addressed: hicks13@llnl.gov
}

depends on the integrated details of the entire ablation process including the flux and spectrum of $\mathrm{x}$ rays produced in the hohlraum, radiation transport to the ablation surface, and the spectral opacities of the ablator before and after blow-off, where conditions throughout may or may not be in local thermodynamic equilibrium. Since it is impossible to guarantee that all these processes can be simulated to the accuracy necessary to achieve ignition the most practical approach is to directly measure both the velocity and mass of the ablator. These observables can then be experimentally tuned by adjusting the initial thickness of the ablator and the power in the peak of the drive to converge iteratively upon the desired conditions. ${ }^{6,7}$ Nominally the velocity needs to be measured to $\sim \pm 2 \%$ while the remaining mass needs to be measured to $\sim \pm 1.5 \%$ of the initial mass. Up until now, however, it has been challenging to measure the capsule velocity and impossible to measure the ablator mass.

Streaked or gated $\mathrm{x}$-ray radiography has long been applied to ICF experiments ${ }^{8-10}$ but the wealth of information encoded in this simple record (Fig. 1) has yet to be fully extracted. In principle, the inverse Abel transform of a radiograph through a spherically-symmetric target can be used to extract the density profile, $\rho(r)$, of the capsule ablator. Knowing this 1-D field quantity at various times $(\rho(r, t))$ then allows multiple 0 -D or average ablator parameters to be calculated from radial moments of $\rho(r, t)$, in particular the average radius, $\langle R(t)\rangle$, mass $M(t)$, areal density $\langle\rho R(t)\rangle$, and average velocity $\langle U(t)\rangle{ }^{11}$ All these 0 -D quantities are valuable figuresof-merit assessing the performance of an ICF implosion. In fact, during much of the implosion 1-D capsule dynamics reduces ${ }^{12}$ to the simple case of a spherical rocket whose only parameters are $\langle R(t)\rangle,\langle U(t)\rangle$, and $M(t)$. A single, streaked radiograph thus contains all the necessary information to describe the global dynamics of the 
spherically-symmetric imploding rocket.

There are several reasons why, until now, this information has yet to be fully extracted from ICF radiographs. The intensity of laser-plasma produced area backlighters, $I_{0}(r, t)$, varies both spatially and temporally, making it difficult to measure the optical depth of the sample. ${ }^{13}$ This problem is compounded by x-ray attenuation in the ablator blowoff, further modifying $I_{0}(r, t)$ spatially and temporally. In addition, capsule radiographs are typically noisy - due to photon statistics, backgrounds, and streak camera noise - presenting challenges for the illconditioned inverse Abel transform.

Here we show that in the specific circumstances of an ICF radiography experiment the problem of an unknown $I_{0}(r, t)$ can be solved by applying two key constraints: (1) A localized ablator density profile in radius space, and (2) A delocalized, or smoothly varying, backlighter intensity profile in object space. We solve the ill-posed and ill-conditioned radiography problem containing an unknown backlighter intensity and noisy data by using a regularization approach that chooses from the ensemble of possible $\rho(r)$ profiles the solution that best obeys these two physically plausible a priori constraints. These constraints quantify what our eyes naturally do: separate sharp variations caused by capsule absorption from those caused by the slowly-varying backlighter and ablator blow-off profile.

The layout of this paper starts with the definitions of certain capsule parameters as moments of $\rho(r)$ (Section II) and summarizes the challenges in ICF radiography of determining $\rho(r)$ from the standard deterministic inverse Abel transform approach (Section III). The regularized Abel transform solution to these problems is described in detail in Section IV along with its generalization to heterogeneous targets such as the gradeddoped ablator (Section V). Since the success of this technique hinges on the validity of various assumptions considerable attention is given to justify these assumptions in terms of intuitive physical descriptions, mathematical proofs where possible, and, most critically, from testing it against simulated data (Section VI). How the results can be used to determine rocket model parameters is then described in Section VII. Finally, the implementation of this technique on OMEGA experiments in discussed (Section VIII).

\section{AVERAGE ABLATOR QUANTITIES AS MOMENTS OF $\rho(r)$}

Several important ablator quantities are defined in terms of moments of $\rho(r)$. In particular the areal density, $\langle\rho \mathrm{R}\rangle$, is given by the zeroth moment, the average radius, $\langle\mathrm{R}\rangle$, by the ratio of the first and zeroth moments, and the mass, $M$, by the second moment:

$$
\langle\rho \mathrm{R}\rangle=\int^{a} \rho(r) \mathrm{d} r
$$

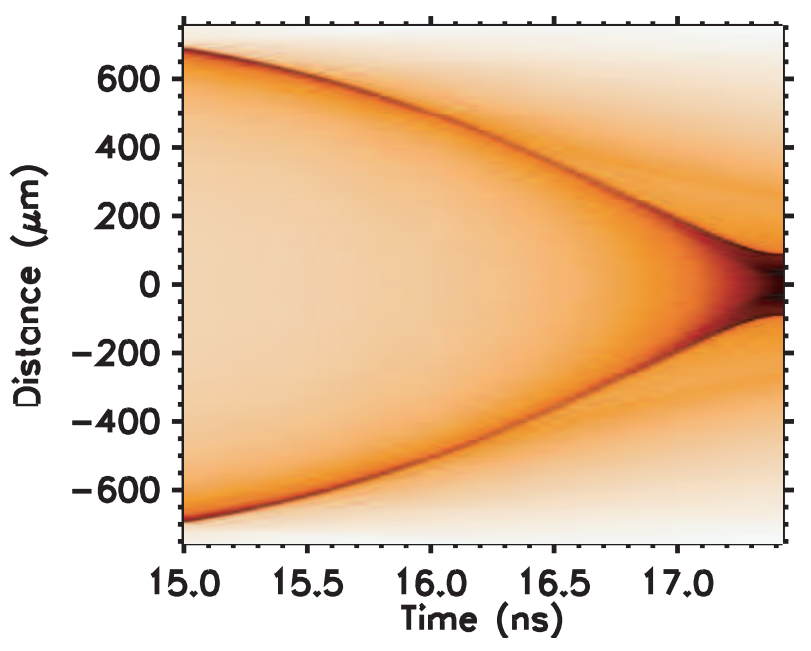

FIG. 1. (Color online) Simulated x-ray streak image of a NIF capsule ${ }^{14}$ implosion made by post-processing a radiationhydrodynamic simulation. Shown is the region where the capsule achieves maximum velocity. This image, along with blurred and noisy versions of it, are used to test for accuracy and precision of the analysis technique.

$$
\begin{aligned}
\langle\mathrm{R}\rangle & =\frac{\int^{a} r \rho(r) \mathrm{d} r}{\langle\rho \mathrm{R}\rangle} \\
M & =4 \pi \int^{a} r^{2} \rho(r) \mathrm{d} r \\
& =4 \pi\langle\rho \mathrm{R}\rangle\left\langle\mathrm{R}^{2}\right\rangle
\end{aligned}
$$

where $\left\langle\mathrm{R}^{2}\right\rangle=\int^{a} r^{2} \rho(r) \mathrm{d} r /\langle\rho \mathrm{R}\rangle$. Here the lower limit of the integrals is the inner radius of the ablator (or $r=0$ if there is no fuel) and the upper limit, $a$, is the position of the ablation front.

Rather than using $\langle\mathrm{R}\rangle$ as defined above it is often useful to define the center of mass:

$$
\left\langle\mathrm{R}_{m}\right\rangle=\frac{\int r \rho(r) r^{2} \mathrm{~d} r}{\int \rho(r) r^{2} \mathrm{~d} r}
$$

Simulations show that $\left\langle\mathrm{R}_{m}\right\rangle \simeq\langle\mathrm{R}\rangle$ over most of the implosion trajectory but that the time derivative of $\left\langle R_{m}\right\rangle$ is better behaved near stagnation.

The mass can more usefully be expressed as:

$$
M=4 \pi\langle\rho \mathrm{R}\rangle\left(\langle\mathrm{R}\rangle^{2}+\left\langle\sigma^{2}\right\rangle\right)
$$

since the variance is $\left\langle\sigma^{2}\right\rangle=\left\langle\mathrm{R}^{2}\right\rangle-\langle\mathrm{R}\rangle^{2}$. For the acceleration phase of an implosion it is usually true that $\left\langle\sigma^{2}\right\rangle \ll\langle R\rangle^{2}$ in which case $\langle\rho \mathrm{R}\rangle$ and $M$ are directly related, regardless of the shell thickness, for a fixed $\langle\mathrm{R}\rangle$.

Since the goal of this measurement is to determine $M(t)$ and $\langle R(t)\rangle$ (and thus $\langle U(t)\rangle=\mathrm{d}\langle R\rangle / \mathrm{d} t$ ), details of $\rho(r)$ are important only insofar as they affect its first three moments. Thus fine scale structure in $\rho(r)$ is unimportant to the extent that it does not significantly affect these moments. This relaxes the requirement on diagnostic spatial resolution and is in part why the regularization 
approach described in Section IV using smoothed density profiles is successful. Determining $\rho(r, t)$ accurately is more challenging from an instrument requirement perspective than is determining its first few moments.

\section{THE INVERSE ABEL TRANSFORM METHOD AND ITS LIMITATIONS}

For spherically-symmetric objects the inverse problem of tomography reduces to finding solutions of the Abel transform. In this section the well-known formulae of absorption radiography and the Abel transform are summarized and used to illustrate how the challenges of an ICF experiment make this classic problem ill-posed. This makes an explicit solution via the inverse Abel transform impossible without additional information.

For standard absorption contrast radiography the observed x-ray intensity, $I(y)$, along a measured dimension, $y$, where $y=0$ corresponds to the center of the object with spherical symmetry, is given by:

$$
I(y) / I_{0}(y)=\exp [-\tau(y)]
$$

where $I_{0}(y)$ is the initial x-ray intensity and $\tau(y)$ is the optical depth along the line of sight.

The forward Abel transform relates this projected optical depth to the product of the object's opacity profile at the backlighter photon energy, $\kappa_{\nu}(r)$, and density profile, $\rho(r)$, where both are functions of radius:

$$
\tau(y)=2 \int_{y}^{\infty} \frac{\kappa_{\nu}(r) \rho(r) r}{\sqrt{r^{2}-y^{2}}} \mathrm{~d} r
$$

The inverse Abel transform is given by:

$$
\kappa_{\nu}(r) \rho(r)=-\frac{1}{\pi} \int_{r}^{\infty} \frac{\mathrm{d} \tau(y)}{\mathrm{d} y} \frac{\mathrm{d} y}{\sqrt{y^{2}-r^{2}}}
$$

Thus a measurement of $\tau(y)$ allows $\rho(r)$ to be determined explicitly provided that $\kappa_{\nu}(r)$ is known.

In a typical streaked radiography experiment of an imploding capsule it is difficult to extract $\rho(r)$ explicitly in this fashion because there are too many unknowns. Firstly, $I_{0}(y)$ in Eq. 7 is unknown. For area backlighting ${ }^{13}$ the spatial and temporal variations in this illumination profile cannot be ignored and are difficult to measure independently. Also, for mid-Z doped capsules there is sufficient opacity in the ablated plasma to further alter the effective $I_{0}(y)$. Secondly, a radially inhomogeneous ablator means that $\kappa_{\nu}(r)$ is unknown. NIF capsule designs use a graded ablator dopant ${ }^{15}$ which causes the unablated $\kappa_{\nu}(r)$ in Eq. 9 to vary throughout the implosion. An additional challenge is that implosion radiographs are particularly noisy because of photon statistics, backgrounds, and camera noise. Regardless of the numerical technique used the inverse Abel transform amplifies noise. ${ }^{16}$

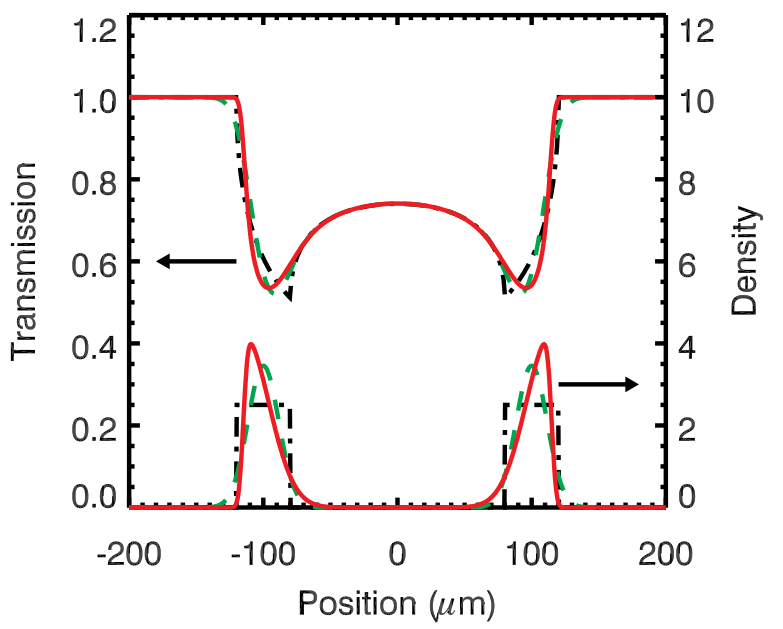

FIG. 2. (Color online) For a spherical shell the x-ray transmission profile is determined largely by the first 3 moments of density. To demonstrate this, transmission profiles are plotted for three different density profiles which have the same $\langle\rho \mathrm{R}\rangle$, $\langle\mathrm{R}\rangle$, and $\left\langle\sigma^{2}\right\rangle$. Even though these density profiles have significantly different peak values the differences in transmission are small and confined to the region around the limb minimum ( $\sim 100 \mu \mathrm{m}$ in this case). Inside the capsule, transmission profiles are nearly identical as required by the Taylor expansion given in Eq. 10 which is independent of higher moments.

\section{A REGULARIZATION APPROACH TO FINDING $\rho(r)$}

\section{A. A priori constraints}

Regularization is a technique used to solve ill-posed or ill-conditioned problems by introducing additional information as constraints. ${ }^{17}$ These constraints reduce the number of unknowns (useful for ill-posed problems) and penalize complexity (useful for ill-conditioned problems). Such a priori constraints, or Bayesian priors, are most valuable when derived from specific knowledge of the problem. The simplest example of regularization is least squares curve-fitting.

Here, for the case of an implosion radiograph, the choice of constraints attempts to capture how the eye instinctively identifies the capsule limb, namely to search for a unique, strongly-localized feature in an otherwise smoothly-varying background. To quantify this distinction between spatial variations caused by capsule absorption and spatial variations caused by backlighter nonuniformities the following a priori constraints on $\rho(r)$ and $I_{0}(y)$ are invoked:

A: That the capsule density profile has compact support. Specifically $\rho(r)=0$ for $r<r_{\min }$ and $r>r_{\max }$ where $r_{\min }>0$ and $r_{\max }$ is less than the maximum radius recorded in the radiograph. Note this is not a thin shell approximation: the shell need not be 
thin, it just needs to be a shell. During the acceleration phase of the implosion this should be the case. For simplicity $\rho(r)$ should also be smooth, i.e. the density profile is a bump function.

B: That the backlighter intensity profile varies over a spatial scale that is larger than the shell thickness. Experimentally this is satisfied by using a large enough backlighter laser focal spot diameter or by suitably overlapping smaller spots.

Importantly, these constraints also remove the effect of unknown attenuation by ablator blow-off. Such absorption is significant in NIF capsules because of the large amount of ablator mass removed, the presence of a mid$\mathrm{Z}$ dopant, and the fact that at high implosion velocities material continues to converge immediately after ablation. As long as the scale length of this ablated material $(>200 \mu \mathrm{m})$ is larger than that of the limb $(\sim 20 \mu \mathrm{m})$ the above assumptions automatically include this additional $\mathrm{x}$-ray attenuation as part of the spatial variation of the backlighter. The ablated material is eliminated from the accounting by deriving an effective $I_{0}(y)$ appropriate for characterizing the unablated material only. Thus the fundamental distinction made between the unablated and ablated material is one of scale length.

The current incarnation of this regularization procedure uses curve fits to constrain the form of $\rho(r)$ and $I_{0}(y)$. Functional forms for $\rho(r)$ that have been tested include a rectangular profile and Gaussian profiles with various degrees of skew (see Fig. 2). For $I_{0}(y)$ polynomials of order 2 to 6 have been tested. As will be described below, the solution is quite insensitive to the choice of these functional forms as long as they obey the two criteria above. This suggests there exists a more generalized mathematical approach to solving this problem than the one reported here.

\section{B. Analysis procedure}

Having set these a priori constraints by choosing the functional forms for $\rho(r)$ and $I_{0}(y)$ the following iterative procedure is used to find $\rho(r)$ at a single time step:

1. Guess the three parameters which fully describe the $\rho(r)$ bump function. For a Gaussian these correspond to the average position, the peak density, and the width. No more than three parameters are used to fully describe the bump function since this is sufficient to define the first three moments of the distribution (if a skewed Gaussian distribution is used the skew is not allowed to vary).

2. For this $\rho(r)$ determine the opacity profile, $\kappa_{\nu}(r)$, by converting from its value in Lagrangian coordinates at $t=0$. This step will be described in Section VD.
3. Using this $\kappa_{\nu}(r) \rho(r)$ profile perform the forward Abel transform to determine the optical depth, $\tau(y)$, of the object (Eq. 8). Here a 3-point numerical forward Abel transform has been used, the inverse of which was found to be more accurate than other numerical integration algorithms. ${ }^{16}$ Since the Abel integral is linear it can be discretized into a linear transformation matrix dependent only on the grid spacing, not on $\kappa_{\nu}(r) \rho(r)$. Calculated once in an expensive step at the start of the problem this matrix can then be efficiently used for all subsequent calculations during each iteration and at each subsequent time step.

4. Convolve $\exp (-\tau(y))$ with the necessary instrument broadening and then recover the 'broadened' $\tau(y)$. Being able to do this is another advantage to performing the forward transform rather than its inverse.

5. Combine this instrument broadened $\tau(y)$ with the measured profile $I(y)$ to determine the effective backlighter profile using $\ln I_{0}(y)=\ln I(y)+\tau(y)$ (from a re-arrangement of Eq. 7).

6. Fit this effective backlighter distribution, $I_{0}(y)$, to a smooth profile. Return to (1) and repeat this loop to minimize the $\chi^{2}$ on this fit. The result is the $\rho(r)$ which is most consistent with a smoothly varying background profile.

This procedure converges on $\rho(r)$ usually in a few iterations. The input parameters for $\rho(r)$ are then used to start the iteration afresh for next time step. In this way only one set of initial guesses need be provided to analyze an entire streaked radiograph. ${ }^{18}$

A fundamental aspect of this analysis is that the two a priori constraints are seemingly opposite to each other and are applied on either side of the Abel transform. The density profile is constrained to be a localized bump function in $r$-space while the backlighter profile is constrained to be a de-localized, smooth function in $y$-space. This notion of applying different constraints alternately in two transformed spaces has parallels in the Gerchberg-Saxton approach for analyzing coherent diffractive images where constraints are applied to real and Fourier spaces. ${ }^{19}$

\section{Justification for form of $\rho(r)$}

Constraining $\rho(r)$ to be of a particular functional form may appear at first to be overly severe. Here justifications are given as to why higher order details of $\rho(r)$ are not very important when the final goal is only to find its first few moments (see also Fig. 2). 


\section{Taylor expansion of the optical depth in terms of density moments}

Inside a shell, where $\rho(r)=0$, the Abel integral Eq. 8 can be Taylor expanded about $r=0$ in terms of the first few moments of $\rho(r)$ :

$$
\tau(y) \simeq \frac{2\left\langle\kappa_{\nu} \rho R\right\rangle}{\sqrt{1-(y /\langle R\rangle)^{2}}}\left[1+\frac{3}{2} \frac{(y /\langle R\rangle)^{2}}{\left(1-(y /\langle R\rangle)^{2}\right)^{2}} \frac{\left\langle\sigma^{2}\right\rangle}{\langle R\rangle^{2}}\right]
$$

The optical depth inside the shell is thus, to a good approximation, only a function of the first three moments, and most sensitive to the first two. This is demonstrated in Fig. 2 where the x-ray transmission is compared for different density profiles with the same first three moments.

This means that $\rho(r)$ can have any number of different functional forms but to match the optical depth profile inside the shell it is only the value of the first few moments that matters.

\section{Moments of $\tau(y)$ and $\rho(r)$}

Another way to appreciate how higher order details in the $\rho(r)$ profile are not essential to determining $\langle\rho R\rangle$, $\langle R\rangle$, and $M$ is to cast the moments of $\rho(r)$ in terms of moments of $\tau(y)$. With some effort it can be shown that:

$$
\begin{gathered}
\tau(0)=2 \int_{0}^{\infty} \kappa_{\nu} \rho(r) \mathrm{d} r=2\left\langle\kappa_{\nu} \rho R\right\rangle \\
\int_{0}^{\infty} \tau(y) \mathrm{d} y=\pi \int_{0}^{\infty} \kappa_{\nu} \rho(r) r \mathrm{~d} r=\pi\left\langle\kappa_{\nu} \rho R\right\rangle\langle R\rangle \\
\int_{0}^{\infty} \tau(y) y \mathrm{~d} y=2 \int_{0}^{\infty} \kappa_{\nu} \rho(r) r^{2} \mathrm{~d} r=\left\langle\kappa_{\nu} M\right\rangle / 2 \pi
\end{gathered}
$$

This demonstrates that in fitting $\tau(y)$ using an assumed functional form of $\rho(r)$ it is only important to capture $\tau(0)$ and the zeroth and first moments of $\tau(y)$ if all that is needed is the zeroth, first, and second moments of $\rho(r)$. Since there are many forms of $\rho(r)$ that can match these conditions the simplest reasonable form should be selected. $\rho(r)$ should be no more complicated than what is necessary to fit these first moments of $\tau(y)$. Higher order moments of $\rho(r)$ do not matter.

Intriguingly, these identities show that Abel transformations are not required if all that is needed are moments of $\rho(r)$. Since it is mostly integrals of $\tau(y)$ that are needed this explains why $\rho(r)$ moments can be determined even with quite noisy and blurred data. This is a useful notion to keep in mind. In this study the Abel transform is still needed, however, in order to (i) impose the constraint of compact support on $\rho(r)$ and to (ii) find $\kappa_{\nu}(r)$, as described next.

\section{OPACITY OF THE UNABLATED MASS}

In general a single radiography measurement on its own has only enough information to determine the density-opacity product, $\kappa_{\nu}(r) \rho(r)$, as given in Eq. 9 . Finding $\rho(r)$ itself requires additional information. This is a particularly important issue for capsules with a graded dopant profile ${ }^{15}$ where the remaining specific opacity of the shell evolves as successive layers get ablated.

Here we show how $\kappa_{\nu}(r)$ can be determined directly from the opacity profile in Lagrangian coordinates using the assumption that mass is ablated from outer regions of the capsule first. This analysis needs no further information if the following approximations are valid: (i) The backlighter $\mathrm{x}$ rays are quasi-monochromatic, (ii) Cold opacities are applicable in the unablated region, and (iii) Mixing of the layers is negligible. These are good starting approximations and can be relaxed as more information becomes available. For example we show how the presence of mix changes the results.

\section{A. Validity of quasi-monochromaticity}

The constraint on spectral bandwidth is relatively modest and is set by Beer's Law (Eq. 7). This equation is valid provided that $\tau_{\nu}-\left\langle\tau_{\nu}\right\rangle \ll 1$ where $\tau_{\nu}$ is the optical depth for available photon energies and $\left\langle\tau_{\nu}\right\rangle$ is the spectrally-averaged optical depth. Under this constraint,

$$
I(y) / I_{0}(y)=\exp \left[-\left\langle\tau_{\nu}(y)\right\rangle\right]=\exp \left[-\sum f_{\nu} \tau_{\nu}(y)\right]
$$

where $f_{\nu}$ is the fraction of photons at a given energy. Thus a quasi-monochromatic spectrum can still be treated as monochromatic provided a spectrally-weighted opacity $\sum f_{\nu} \kappa_{\nu}$ is used. X-ray He- $\alpha$ fluorescence spectra generated by laser intensities of $\sim 10^{14}-10^{15} \mathrm{~W} / \mathrm{cm}^{2}$, as are used in this study, have been well documented previously ${ }^{20}$ and are sufficiently monochromatic for optical depths of 1-2.

\section{B. Validity of cold opacities}

Use of cold opacities simplifies the analysis considerably since this makes $\kappa_{\nu}$ independent of temperature and density. Opacity model calculations ${ }^{21}$ at photon energies of $6-10 \mathrm{keV}$ show that in local thermodynamic equilibrium cold opacities are valid in beryllium, carbon, copper, and germanium for temperatures below $50 \mathrm{eV}, 90$ $\mathrm{eV}, 150 \mathrm{eV}$, and $300 \mathrm{eV}$ respectively. These thresholds increase with density. Since temperatures in the unablated portions of the NIF capsule are expected to stay below $60 \mathrm{eV}$ during the acceleration phase cold opacities are generally a good approximation.

Upon ablation opacities drop due to ionization of the K-shell (in the case of beryllium and carbon) or the Lshell (in the case of copper and germanium). This provides a natural distinction between the ablated and unablated region since x-ray absorption occurs primarily in the unablated material. While this is a convenient 
$15.5 \mathrm{~ns}$

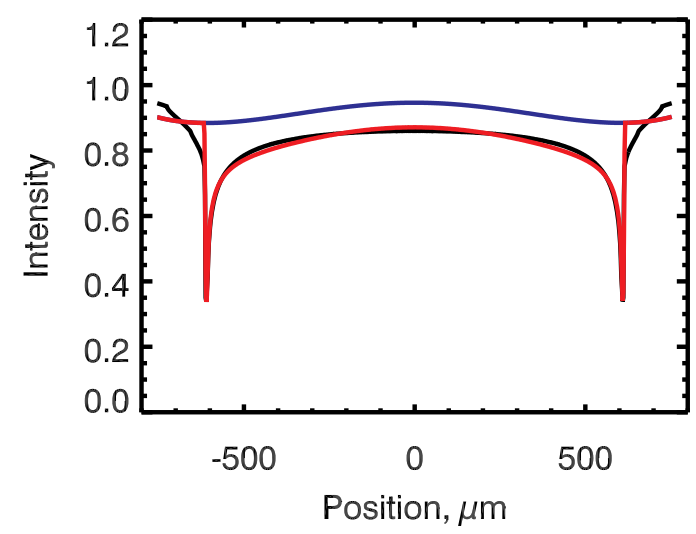

$16.5 \mathrm{~ns}$

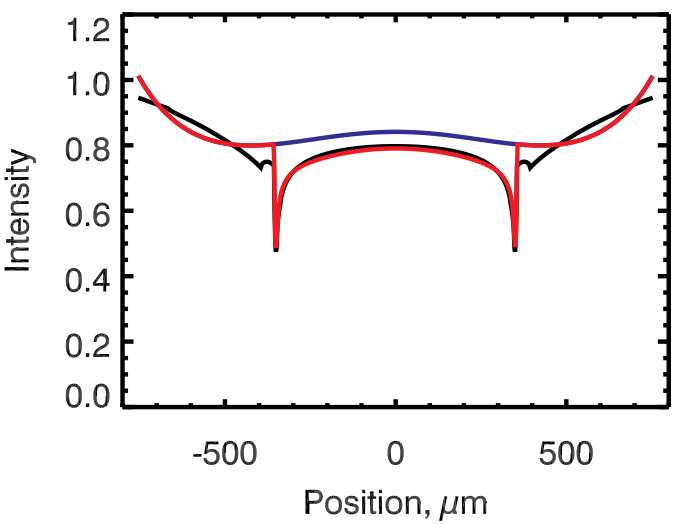

$16.0 \mathrm{~ns}$
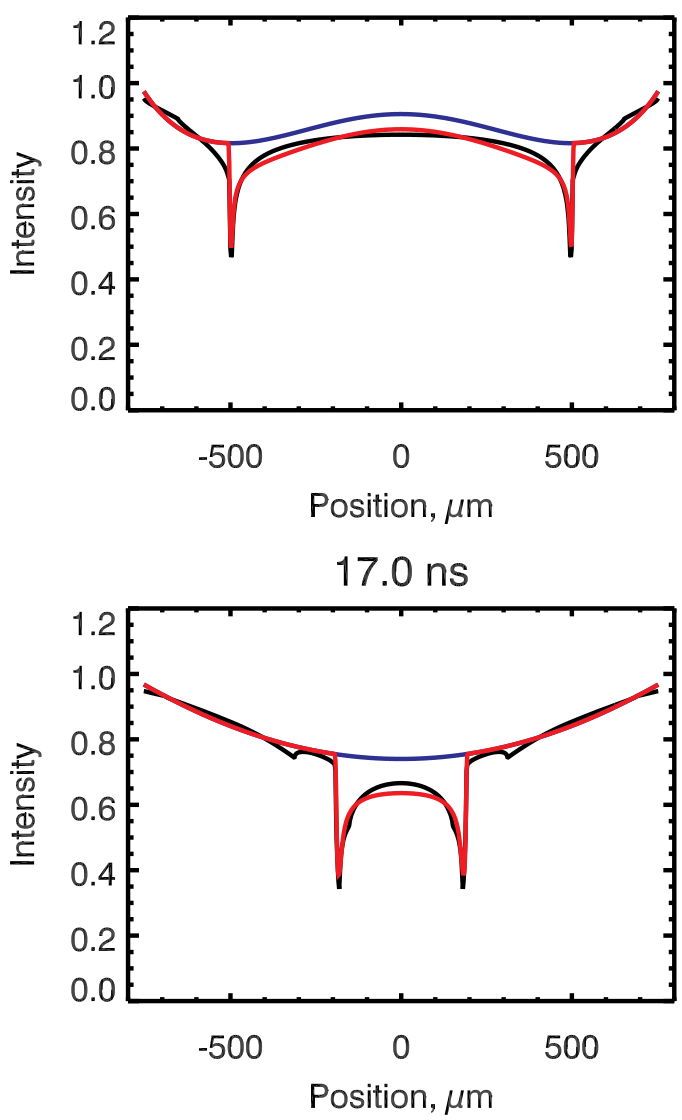

FIG. 3. (Color) Simulated x-ray intensity profile at various times (black) along with the best fit from the regularization analysis (red) and the inferred unattenuated intensity (blue). In this particular analysis a skewed Gaussian was used for $\rho(r)$ and a 4 th order polynomial for $I_{0}(y)$. No spatial or temporal blurring in the simulation or the analysis was used in this test.

feature for a diagnostic designed to detect only the unablated shell it is actually the scale length of the limb, as described in Section IV A, that distinguishes unablated from ablated material.

\section{Opacity of a multi-component ablator}

The presence and distribution of different elements in the ablator must be properly taken into account in any opacity calculation since even trace amounts of a higher$\mathrm{Z}$ element can affect the average opacity. We show here for completeness how we calculate the average opacity in a multi-component ablator.

At any given position, $r$, inside the capsule the density is given by the sum of the partial densities for each of the elements. Thus,

$$
\rho(r)=\sum_{i} \frac{A_{i}}{N_{A}} n_{i}(r)=\frac{\sum_{i} n_{i}(r)}{N_{A}} \sum_{i} A_{i} F_{i}(r)
$$

where $F_{i}(r)=n_{i}(r) / \sum_{i} n_{i}(r)$ is the atom fraction for each element $i$ at that radius, $n_{i}(r)$ is the atomic number density, $A_{i}$ is the mass number, and $N_{A}$ is Avogadro's Number.

If the initial density profile in the capsule is known from metrology the quantity $\sum_{i} n_{i}(r) / N_{A}$ can be determined directly. Otherwise a reasonable estimate of $\rho(r)$ for different dopant levels can be obtained by assuming that $\sum_{i} n_{i}$ is constant throughout the ablator, regardless of dopant concentration (i.e. the volume per atom is constant). Then, given a known un-doped density, $\rho_{U}$, the density in a doped region is given by $\rho_{D}=\rho_{U} \sum_{i} A_{i} F_{i} /\left(\sum_{i} A_{i} F_{i}\right)_{U}$.

The opacity-density product relevant to radiography measurements is then:

$$
\begin{aligned}
\sum_{i} \kappa_{\nu i}(r) \rho_{i}(r) & =\frac{\sum_{i} n_{i}(r)}{N_{A}} \sum_{i} \kappa_{\nu i} A_{i} F_{i}(r) \\
& =\rho(r) \frac{\sum_{i} \kappa_{\nu i} A_{i} F_{i}(r)}{\sum_{i} A_{i} F_{i}(r)}
\end{aligned}
$$

allowing the average opacity at a given radius to be defined as:

$$
\kappa_{\nu}(r)=\frac{\sum_{i} \kappa_{\nu i} A_{i} F_{i}(r)}{\sum_{i} A_{i} F_{i}(r)}
$$


Ultimately to proceed with the analysis it is necessary to know $\rho(r)$ and $\kappa_{\nu}(r)$ for the initial target. This allows the opacity profile in Lagrangian coordinates, $\kappa_{\nu}(m)$, to be calculated and used as described next.

\section{Determining $\kappa_{\nu}(r)$ by a transformation from Lagrangian coordinates}

The heterogeneous ablator complicates analysis of the radiograph since the opacity profile $\kappa_{\nu}(r)$ is constantly changing as material is ablated. Determining $\kappa_{\nu}(r)$ at each time step can still be done however by recognizing that the opacity profile for the unablated shell is time invariant in Lagrangian coordinates. Each guess for $\rho(r)$ made in the iterative loop (Section IV B) provides the necessary transformation between Eulerian radius-space and Lagrangian mass-space. Then, given that $\kappa_{\nu}(m)$ for the unablated shell is known (see previous section) and time-invariant, $\kappa_{\nu}(r)$ can readily be calculated for the assumed $\rho(r)$. Any given $\rho(r)$ is associated with a specific $\kappa_{\nu}(r)$ - the two are not independent. This is an advantage of performing the forward Abel transform and iterating about assumed forms of $\rho(r)$ rather than attempting the inverse calculation.

This technique is rigorous in that it assigns the appropriate $\kappa_{\nu}$ to each mass element in $\rho(r)$. An approximation that is computationally more efficient and has essentially identical results is to assume that the opacity of the remaining mass is constant across the entire density profile and equal to the average opacity of the unablated mass. This average opacity is given by

$$
\overline{\kappa_{\nu}}(M)=\frac{\int_{0}^{a} \kappa_{\nu}(m) \mathrm{d} m}{\int_{0}^{a} \mathrm{~d} m}=\frac{\int_{0}^{a} \kappa_{\nu}(r) \rho(r) r^{2} \mathrm{~d} r}{\int_{0}^{a} \rho(r) r^{2} \mathrm{~d} r}
$$

This approximation works because details of the opacity distribution are higher order effects that have little effect on the first few moments of $\kappa_{\nu}(r) \rho(r)$ (see Section IV C).

The assumption of a time-invariant $\kappa_{\nu}(m)$ relies on cold opacities remaining valid for the unablated shell (Section VB) and there being no mix to disrupt the original Lagrangian profile. This latter assumption is discussed next.

\section{E. Effects of mix}

The effect of fine-scale mix can be estimated by convolving the original $\kappa_{\nu}(r)$ over a presumed mix width. How this changes the inferred mass is determined by calculating the quantity $\overline{\kappa_{\nu}}(M)$ given by Eq. 15 . As shown in Figure 4 the deviation of the resulting inferred mass from the real mass depends on the amount of mass remaining. Specifically if the ablation front is positioned near the innermost interface between the undoped and doped layers the deviation is largest since the amount of dopant in the remaining mass has changed significantly.
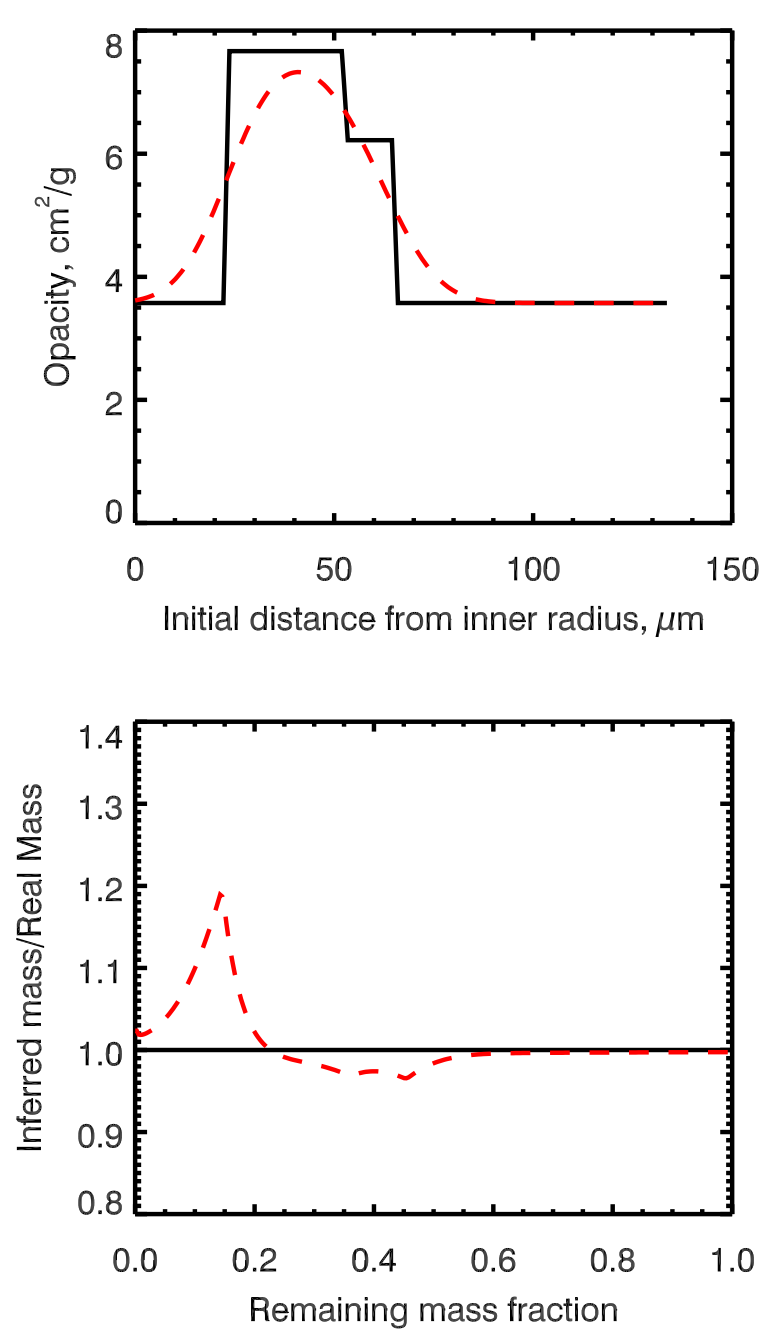

FIG. 4. (Color online) The presence of fine-scale mix will cause the original ${ }^{14}$ opacity profile, $\kappa_{\nu}(r)$ (solid line), to change. In the top figure this original profile is convolved over a $20 \mu \mathrm{m}$ mix region (red dashed line). The resulting deviation in the inferred mass is shown in the bottom figure, where the abscissa corresponds to the remaining mass fraction inside the ablation front. The amount of the deviation depends on the relative opacities of the different layers and the position of the ablation front.

If however the ablation front is in the middle of the doped layer or in the undoped region there is minimal effect since the mix hasn't significantly shifted the total fraction of dopant that exists on either side of the ablation front. This conclusion is dependent upon the mix width chosen. Note that this type of analysis, which assumes a non-radial mixing scale smaller than the diagnostic resolution, cannot address the type of mix where an individual finger or bubble dominates the limb profile. 
$15.5 \mathrm{~ns}$

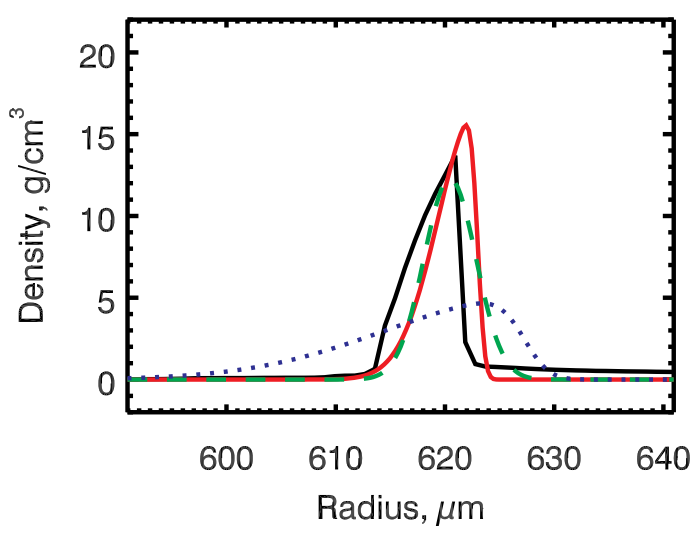

$16.5 \mathrm{~ns}$

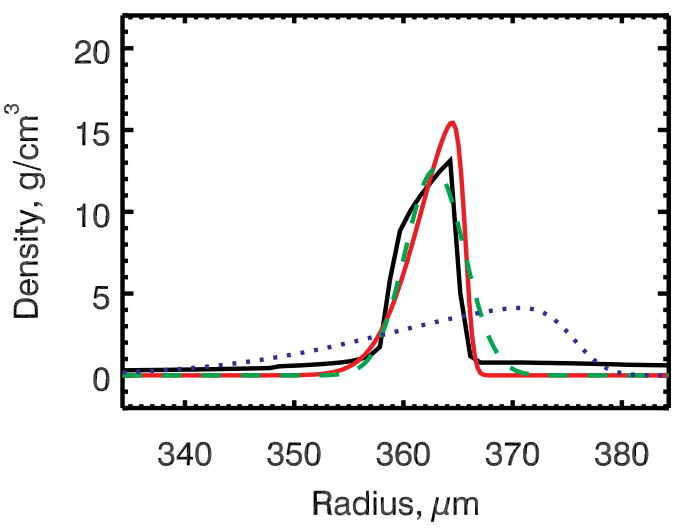

$16.0 \mathrm{~ns}$
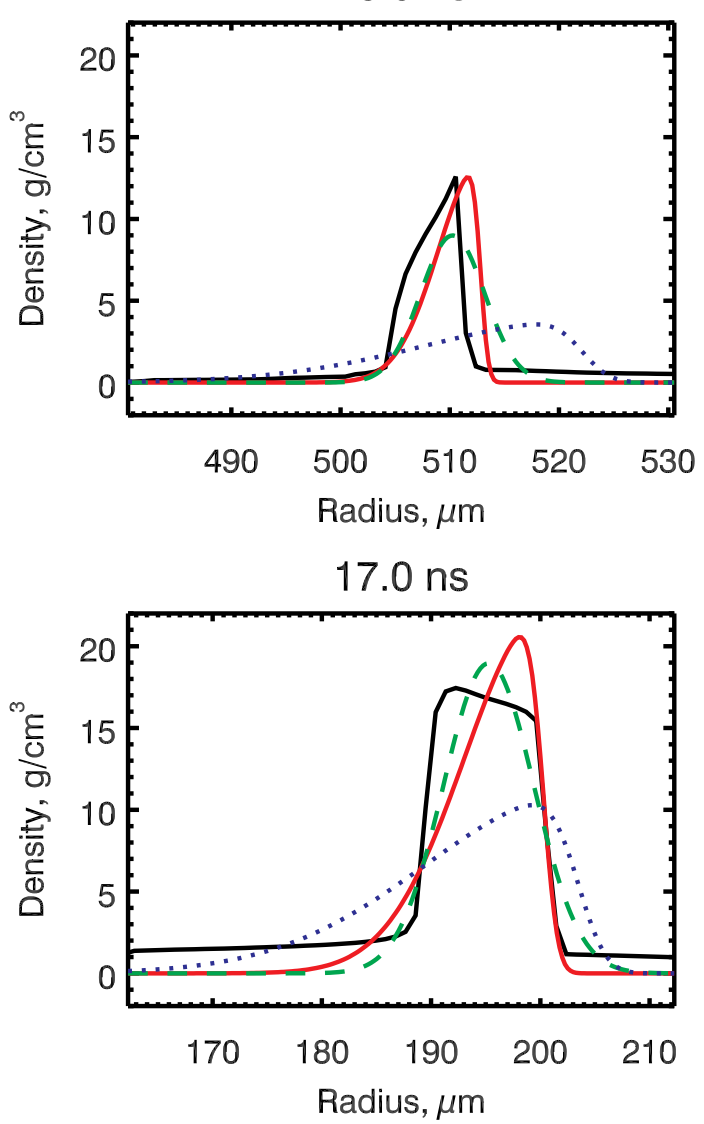

FIG. 5. (Color) Comparison of simulated density profiles (black) with the density profiles inferred using the analysis assuming a skewed Gaussian (red), a symmetric Gaussian (dashed green), and a skewed Gaussian where the x-ray streak had $20 \mu \mathrm{m}$ of spatial and $50 \mathrm{ps}$ of temporal blurring (dotted blue). These density profiles are those for which the best fits were obtained in Fig. 3. Although the inferred profiles, particularly that for the blurred image, appear to deviate from the actual profiles the moments of $\rho(r)$ are still captured accurately (see next Figure).

\section{TESTS ON SIMULATED DATA}

To demonstrate the accuracy and precision of this technique we show in detail the results from analyzing the simulated radiograph of a NIF implosion shown in Fig. 1. This radiograph, taken at the end of the acceleration phase, was generated by forward Abel transforming density profiles calculated using the radiationhydrodynamics code Hyades. ${ }^{22}$ The target - a $134 \mu \mathrm{m}$ thick graded-doped $\mathrm{CH}$ ablator ${ }^{14}$ with a $784 \mu \mathrm{m}$ inner radius - was driven by a shaped radiation source peaking at $285 \mathrm{eV}$ at $16.5 \mathrm{~ns}$. A NIF implosion is a stringent test of the regularization analysis because of the complicated dopant profile, which has to be tracked self-consistently, the large mass of capsule blow-off, which has to be discriminated from the unablated mass, and the high implosion velocity, which causes temporal blurring.

The x-ray intensity profiles using an $8.35 \mathrm{keV} \mathrm{Cu} \mathrm{He-} \alpha$ backlighter are shown in Fig. 3 at four times along with the regularized solutions. In red is the best fit, $I(y)$, while in blue is the inferred backlighter profile, $I_{0}(y)$. As dis- cussed in Section IV A, $I_{0}(y)$ is the apparent backlighter intensity after attenuation by the blow-off plasma. In this example the initial backlighter profile is set to be 1 .

The density profiles found from the analysis are shown in Fig. 5 and compared to the actual density profiles from the simulation. The actual $\rho(r)$ (black) is compared to the regularized profiles found by assuming a skewed Gaussian (red), a symmetric Gaussian (green), and a skewed Gaussian where the image had $20 \mu \mathrm{m}$ of spatial blurring and 50 ps of temporal blurring. Differences between the skewed and symmetric Gaussians are relatively small but the $\rho(r)$ inferred from the blurred image has a low peak density and a large width, as one would expect from a convolution process.

The resulting $\langle R(t)\rangle,\langle U(t)\rangle, \rho R(t)$, and $M(t)$, shown in Fig. 6, are almost always within several percent of the exact value. This is true even for the blurred image despite the spatial convolution width $(20 \mu \mathrm{m})$ being considerably larger than the $2 \sigma$ thickness of the shell $(\sim 5-10$ $\mu \mathrm{m}$ ) over much of the time. Thus blurring has little affect on the extensive, integral properties of $\rho(r)$ important to 

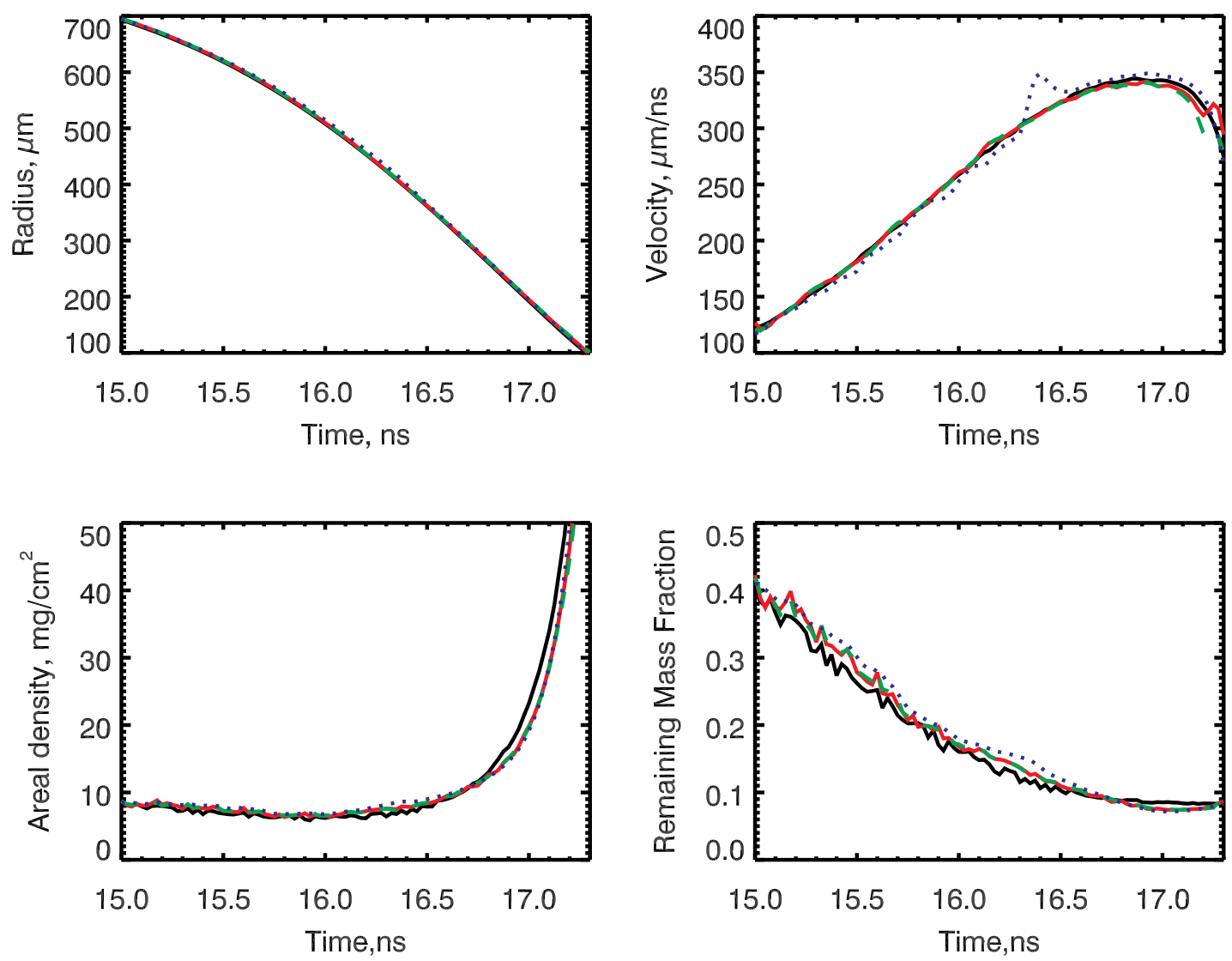

FIG. 6. (Color) Comparison of the simulated $\langle\mathrm{R}\rangle,\langle U\rangle, \rho \mathrm{R}$, and $M$ (black) with those inferred using the analysis assuming a skewed Gaussian (red), a symmetric Gaussian (dashed green), and a skewed Gaussian where the x-ray streak had $20 \mu \mathrm{m}$ of spatial and 50 ps of temporal blurring (dotted blue). These illustrate how little instrumental blurring and the different assumed density profiles affect the results. Note that for the blurred case, even though the spatial blurring is a factor of 2 or more greater than the $2 \sigma$ width of the density profile, agreement (for these average quantities) remains good. The velocity aberration at $16.35 \mathrm{~ns}$ in the blurred case occurs at the boundary between doped and undoped $\mathrm{CH}$.

this study (see Section IV C) but significantly affects how accurately the intensive properties such as peak density can be inferred.

Having established the accuracy of the technique under its various assumptions, the precision of the technique was tested by comparing an ensemble of synthetic static radiographs with different levels of Poisson noise. Not surprisingly the fractional random error in $\langle\mathrm{R}\rangle$ and $M$ scales with $1 / \sqrt{(} N)$ where $N$ is the number of counts per resolution element, as shown in Fig. 7. The fractional random error in $\rho \mathrm{R}$ is identical to that for $M$ and is not shown. The velocity, since it involves a differential, is very sensitive to noise and for noisy data it is often more practical to take the derivative of a fit to $\langle R\rangle$ versus $t$.

\section{ROCKET MODEL ANALYSIS}

Global capsule dynamics simplifies to that of a spherically imploding rocket for much of the implosion history. ${ }^{1,12}$ In the simplest version of this model, valid from the end of the shock compression phase to the period around maximum velocity, ${ }^{12}$ the time dependence of the rocket radius, velocity, and mass is determined purely by the ablation pressure, $p_{a}$, and the mass ablation rate, $\dot{m}_{a}$ :

The dynamics of the rocket are given by:

$$
\begin{aligned}
\frac{\mathrm{d} M}{\mathrm{~d} t} & =-4 \pi R^{2} \dot{m}_{a} \\
M \frac{\mathrm{d}^{2} R}{\mathrm{~d} t^{2}} & =-4 \pi R^{2} p_{a}+f_{b}
\end{aligned}
$$

Here $f_{b}=8 \pi \int_{0}^{R} p r \mathrm{~d} r$ is the decelerating force caused by internal pressure, $p$, in the ablator, fuel, and gas fill. For most of the acceleration phase $f_{b}=0$, resulting in the simple rocket model applied below.

Since data from the streaked radiograph yields $\langle R(t)\rangle$ and $M(t)$ it is possible to extract both $p_{a}$ and $\dot{m}_{a}$ from the data, averaged over a finite time period. This can be done either by directly taking derivatives of the data 


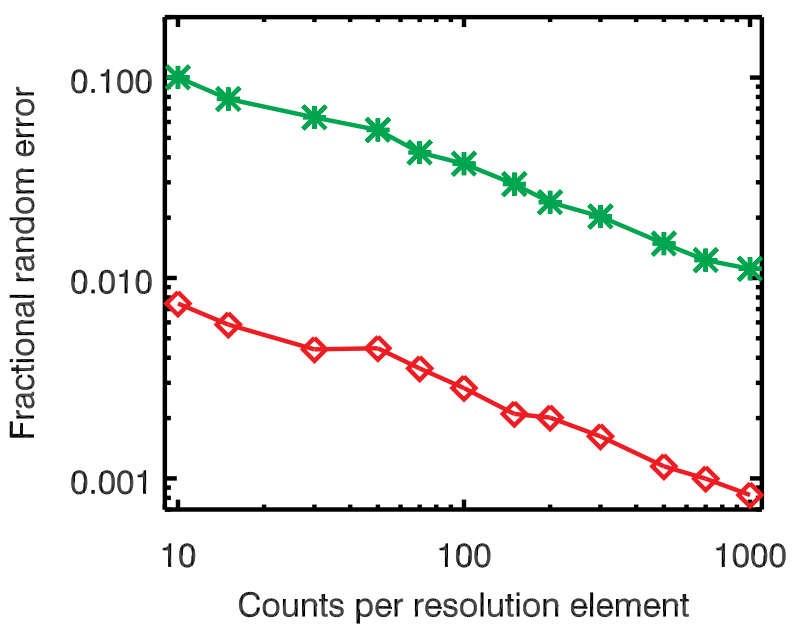

FIG. 7. (Color online) Illustrative scaling of the fractional random error in $\langle\mathrm{R}\rangle$ (diamonds, red) and $M$ (asterisks, green) versus the number of counts per resolution element $(N)$. Each point was derived from the analysis of a synthetic static radiograph with counts based on Poisson statistics. Both $\langle\mathrm{R}\rangle$ and $M$ scale closely with $1 / \sqrt{N}$.

and using the above equations directly (which is difficult when noise is present) or by fitting integrated forms of these rocket equations to the data. The latter is a multivariate orthogonal distance regression ${ }^{23}$ problem requiring fitting of a curve in the 3-dimensional phase space defined by $t, R$, and $M$. Details of this type of analysis will be described elsewhere. Results from the technique, applied to the $R(t)$ and $M(t)$ extracted in Fig. 6, are shown in Fig. 8. The inferred $p_{a}$ and $\dot{m}_{a}$ are very close to the values obtained directly from the radiationhydrodynamics simulation. Late in time, $f_{b}$ can no longer be neglected and the inferred pressure underestimates the ablation pressure.

\section{EXPERIMENTAL TEST}

\section{A. Experimental set-up}

An experimental test of this technique was performed at the OMEGA laser facility, a neodymium-doped phosphate glass system operating with frequency-tripled, 0.35 $\mu \mathrm{m}$ light. ${ }^{25}$ Indirectly-driven implosions were probed using area backlit x-ray streaked radiography with $5.2 \mathrm{keV}$ $\mathrm{x}$ rays from Vanadium He- $\alpha$ fluorescence. A schematic of the experimental setup is shown in Fig. 9.

Copper-doped beryllium capsules with no gas fill were used. The ablators had an inner radius of $215 \mu \mathrm{m}$ and were composed of an inner layer of $4 \mu \mathrm{m}$ pure Be, a $26 \mu \mathrm{m}$ middle layer of beryllium doped with 3.0 atomic percent of $\mathrm{Cu}$, and an outer layer of either 6 or $21 \mu \mathrm{m}$ thick pure Be. Dopant concentrations were chosen so that the
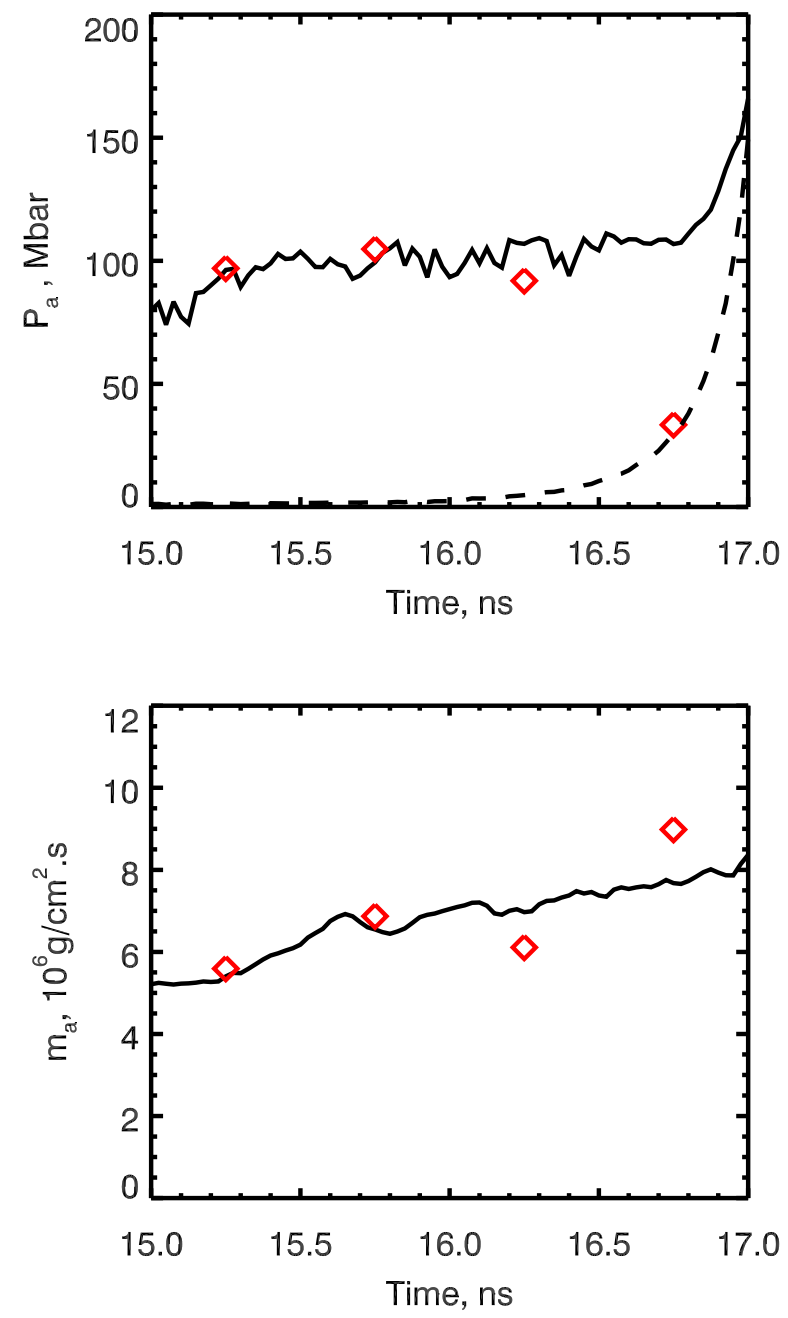

FIG. 8. (Color online) Ablation pressure, $p_{a}$, and mass ablation rate, $\dot{m}_{a}$, for the simulated implosion shown in Fig. 6 . The values obtained by fitting the simple rocket model to the experimental observables $M(t)$ and $R(t)$ are shown as red diamonds and compare favorably with the values obtained directly from the hydrodynamic simulation (black line). Around the time of peak velocity (16.5-17.0 ns) the contribution of the capsule back pressure, $f_{b}$ (dashed line) reduces the accelerating force on the capsule causing an underestimate of the ablation pressure based on the simple rocket model.

optical depths would be comparable to those expected when backlighting NIF capsules using higher backlighter energies.

Each capsule was held inside a hohlraum composed of $25 \mu \mathrm{m}$ thick Au. Hohlraums were $1.6 \mathrm{~mm}$ in diameter and $2.5 \mathrm{~mm}$ long with a $1.2 \mathrm{~mm}$ diameter laser entrance hole (LEH) at each end. No hohlraum gas fill was used. The axis of rotation of the hohlraum was aligned along the P6-P7 axis of the OMEGA target chamber. For radiography access two narrow slots were made on either side of the hohlraum. The direction of the slots was par- 


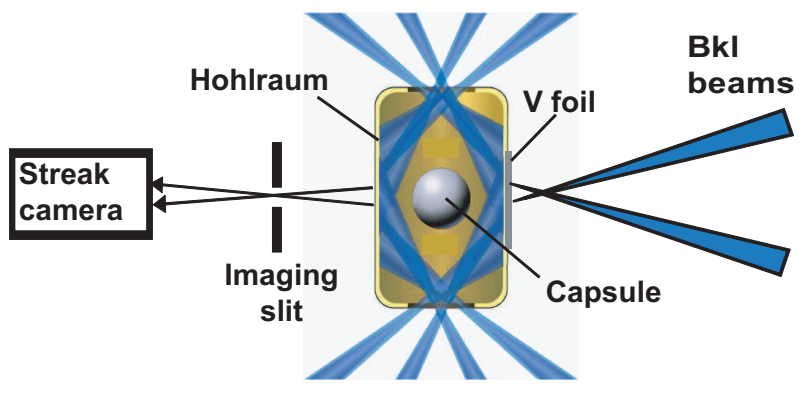

FIG. 9. (Color online) Schematic (not to scale) of the OMEGA experimental setup. The Vanadium foil is glued on to the slot on the backlighter side of the hohlraum. A matching slot on the opposite side of the hohlraum allows $\mathrm{x}$ rays to propagate through the imaging slit to the $\mathrm{x}$-ray streak camera. The imaging slit is positioned to magnify the image by a factor of 20.5 .

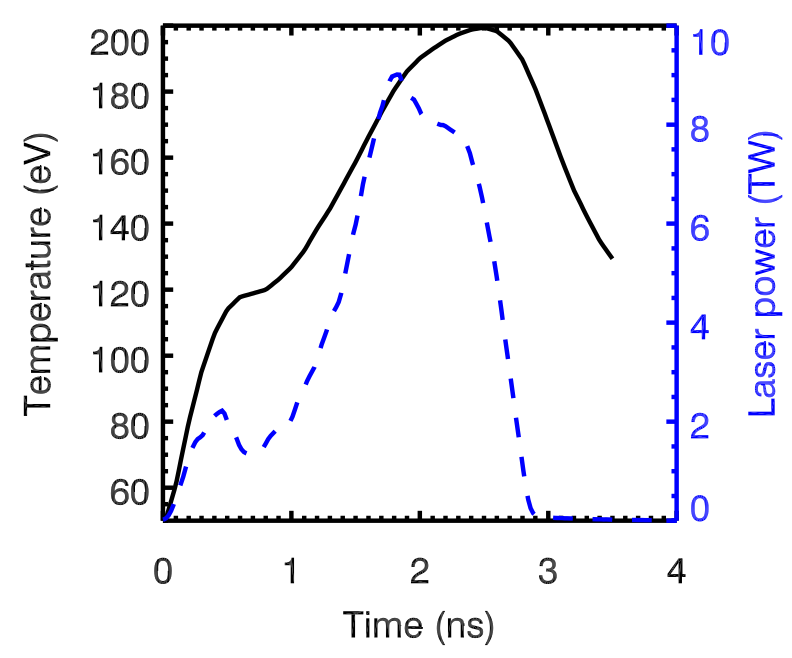

FIG. 10. (Color online) Hohlraum radiation temperature measured using the Dante soft x-ray spectrometer ${ }^{24}$ (solid black line). The total laser power incident on the hohlraum is shown as a blue dashed line.

allel to the hohlraum axis. The slot on the backlighter side (H7) was $1000 \mu \mathrm{m}$ long and $300 \mu \mathrm{m}$ wide while that on the streak camera side (H14) was $1000 \mu \mathrm{m}$ long and $100 \mu \mathrm{m}$ wide. The $\mathrm{H} 7$ slot was covered by a $5 \mu \mathrm{m}$ thick $\mathrm{V}$ backlighter foil while the H14 slot was covered with a $50 \mu \mathrm{m}$ thick $\mathrm{CH}$ foil to prevent closure of the slot during laser ablation of the hohlraum. Slots were offset in opposite directions from hohlraum center to account for the radiography axis (H7-H14) being $10.8^{\circ}$ off-perpendicular from the hohlraum axis. Calibration shots were taken on hohlraums without capsules to measure the backlighter spatial profile and, in conjunction with a grid on the H14 slot, to experimentally measure the magnification.

A total of 40 beams were used to heat the hohlraum. The 20 beams illuminating each LEH were incident at $29^{\circ}$ ( 5 beams), $42^{\circ}$ ( 5 beams), and $59^{\circ}$ (10 beams) to

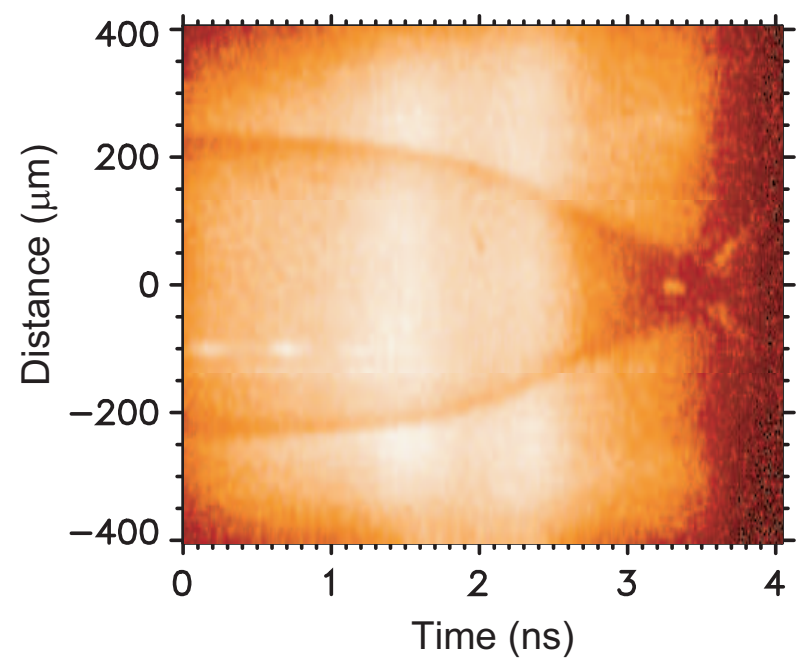

FIG. 11. (Color online) A sample x-ray streak image of an imploding capsule from OMEGA showing the entire time history of the implosion from shock compression to shell acceleration to stagnation at $3.3 \mathrm{~ns}$. The UV timing fiducials are visible at early times.

the hohlraum axis. A shaped laser pulse approximately $2.7 \mathrm{~ns}$ in duration was used (see Fig. 10). Due to facility constraints the same laser pulse shape was used for the backlighter pulses. Maximum available energies of $\sim 320$ $\mathrm{J} /$ beam were used on all shots.

Eight backlighter beams were used to illuminate the central $\sim 800 \mu \mathrm{m}$ field of view of the hohlraum slots. Beams were tiled in space and staggered in time to optimize the x-ray emission brightness and uniformity in both space and time. The beams were incident at $15^{\circ}$, $25^{\circ}, 49^{\circ}$, or $60^{\circ}$ to the backlighter normal, with a pair of beams at each angle. Both $60^{\circ}$ beams were focused to $150 \mu \mathrm{m}$ spots at the backlighter center while one from each of the other pairs was pointed to a $400 \mu \mathrm{m}$ diameter spot centered $150 \mu \mathrm{m}$ to the left or right of backlighter center. The $49^{\circ}, 25^{\circ}$, and $15^{\circ}$ beams began at $-1,0$, and $+1 \mathrm{~ns}$ respectively while one of each of the $60^{\circ}$ beams was timed at 0 or $+1 \mathrm{~ns}$ (here 0 ns represents the time when the heater beams begin).

The primary diagnostic was the SSC-A x-ray streak camera $^{26}$ run with a $\mathrm{Au}$ photocathode. Images were recorded on a $4096 \times 4096$ charge-coupled device using $2 \times 2$ binning. The streak camera slit was $500 \mu \mathrm{m}$ wide and positioned at $49.5 \mathrm{~cm}$ from target chamber center (TCC). The $10 \mu \mathrm{m}$ wide imaging slot was positioned at $2.82 \mathrm{~cm}$ from TCC with a final magnification of 20.5 and a spatial resolution of $\sim 10 \mu \mathrm{m}$. The camera sweep speed was set to capture a sweep window of $\sim 4 \mathrm{~ns}$, giving a time resolution of $\sim 50$ ps. A UV timing comb was used on each streak record to measure the sweep speed and provide and absolute timing reference. The multichannel soft x-ray diagnostic Dante ${ }^{24}$ was run to capture the time history of the hohlraum x-ray emission and thus radiation temperature. 

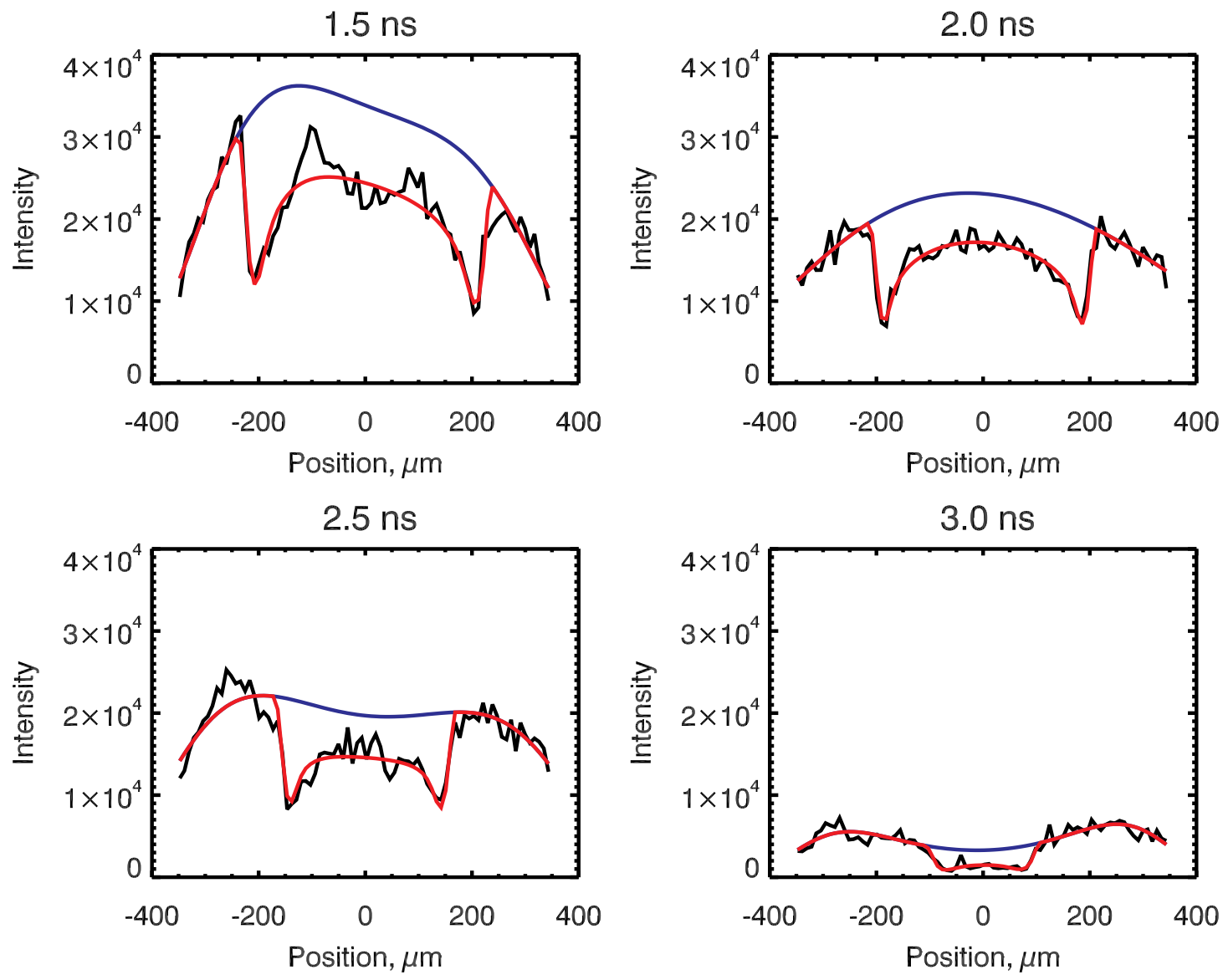

FIG. 12. (Color) Streaked x-ray intensity profile from Fig. 11 at 4 times (black) along with the best fit from the regularization analysis, $I(y)$ (red), and the inferred backlighter intensity, $I_{0}(y)$ (blue). Note how $I_{0}(y)$ evolves in time due to changes in x-ray emission from the backlighter foil and time-evolving attenuation by the ablator blow-off. Data were binned 10 pixels in time and space to reduce noise.

\section{B. Results}

X-ray streaked radiographic images were obtained on shots under similar drive conditions using targets with either $36 \mu \mathrm{m}$ or $51 \mu \mathrm{m}$ thick ablators. A sample streak is given in Fig. 11 for a capsule with a $36 \mu \mathrm{m}$ thick ablator. The measured hohlraum radiation temperature is shown in Fig. 10 and reaches a peak of $200 \mathrm{eV}$.

Sample x-ray intensity profiles from this streak record are shown in Fig. 12 for 4 different times. The best fit intensity profile, $I(y)$, found using the regularization procedure is shown in red with the inferred backlighter intensity, $I_{0}(y)$, shown in blue. As described in Section IV A the inferred shape of $I_{0}(y)$ captures the spatial variation in the backlighter source as well as that caused by the blow-off plasma. The spatial and temporal variation in $I_{0}(y)$ is apparent in the various profiles.

The inferred $\langle R(t)\rangle,\langle U(t)\rangle,\langle\rho R(t)\rangle$, and $M(t)$ for this $36 \mu \mathrm{m}$ thick ablator shot are shown in red in Fig. 13. Data from a target with a $51 \mu \mathrm{m}$ thick ablator is shown in black. Each point without an error bar represents the result from the regularization analysis at a single time step.
Data with error bars give the statistical average of points within successive time windows consisting of $\sim 15$ data points each. Also shown are the results from radiationhydrodynamic ${ }^{27}$ simulations. These calculations are in reasonable agreement with the data over most of the time history of the implosion. A fit of the rocket model to the data around the time of peak radiation drive gives an ablation pressure $P_{a}=79 \mathrm{Mbar}$ and a mass ablation rate $\dot{m}_{a}=4.3 \times 10^{6} \mathrm{~g} / \mathrm{cm}^{2}$.s. This is comparable to that expected from scaling laws at $T_{r}=200 \mathrm{eV} .^{1}$

\section{CONCLUSIONS}

An x-ray streaked radiography technique to measure the time-resolved radius, velocity, $\rho \mathrm{R}$, and mass of imploding capsules has been developed as part of the effort to achieve ignition at the NIF. $\rho(r)$ is extracted from the $\mathrm{x}$-ray transmission profile at each time step with the integrated quantities - $\rho \mathrm{R}$, radius, and mass - then being determined from the first three moments in $\rho(r)$.

Regularization is used to address the ill-posed problem 

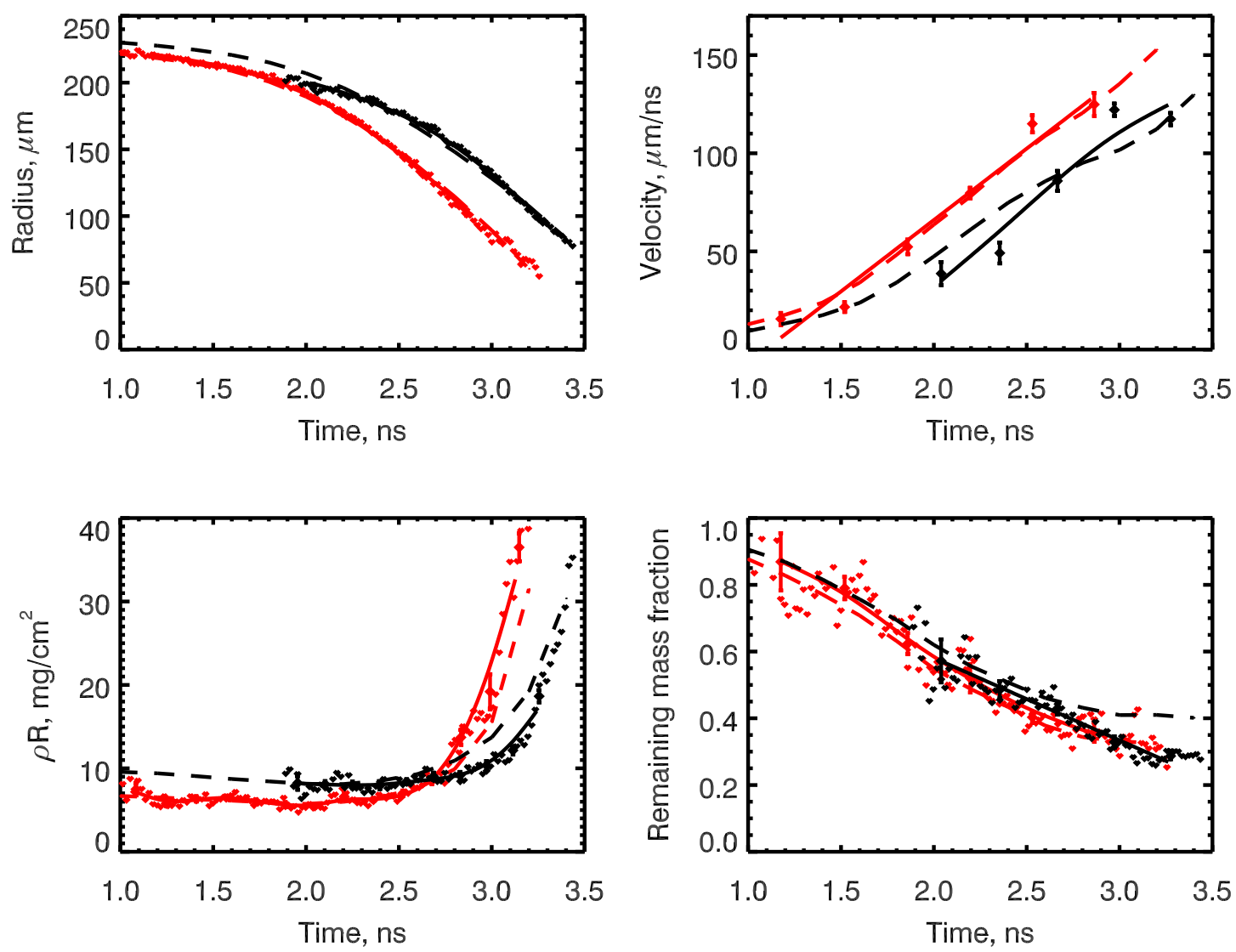

FIG. 13. (Color) Average ablator radius, velocity, $\rho \mathrm{R}$, and remaining mass fraction versus time for targets with either $36 \mu \mathrm{m}$ thick (red) or $51 \mu \mathrm{m}$ thick ablators (black). Data are binned in time allowing statistical uncertainties to be determined from errors in the coefficients of a local linear fit. Binned points (shown with errors bars) are connected by smoothing splines (solid

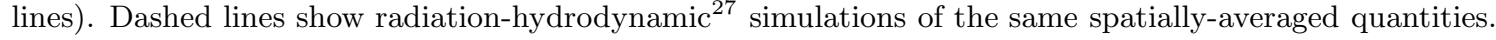

of having an unknown backlighter emission profile and unknown x-ray attenuation in the blow-off plasma. The solution is found to be that which optimally satisfies the a priori constraints that $\rho(r)$ is localized in radius space and $I_{0}(y)$, the effective backlighter profile, is smooth and delocalized in object space. It is thus the scale length that distinguishes shell absorption variations from backlighter variations.

The Bayesian strategy of optimizing a solution subject to a priori constraints means that the technique is only as accurate as the constraints chosen. Three approaches were taken to justifying and validating the technique and its constraints: (i) Mathematical proofs were derived to show that higher moment details of $\rho(r)$ are not important, thus giving freedom to choose the functional form of $\rho(r)$. (ii) A rigorous analysis of a simulated NIF radiograph demonstrated excellent fidelity in tracking the average radius, velocity, $\rho \mathrm{R}$, and mass even when the spatial resolution was poorer than the ablator thickness. (iii) A full experimental test of the technique at the OMEGA laser system showed good agreement with radiation-hydrodynamic simulations. Together these re- sults demonstrate that the technique is ready for use on upcoming experiments at the NIF.

\section{ACKNOWLEDGEMENTS}

We thank the OMEGA operations crew at the Laboratory for Laser Energetics for their efforts during the experiment; A. Nikroo, H. Huang, and K. Moreno at General Atomics and R. Wallace at LLNL for target fabrication; and C. W. Mauche and B. G. Wilson for the hard x-ray opacity calculations. This work was performed under the auspices of the U.S. Department of Energy by Lawrence Livermore National Laboratory under Contract DE-AC52-07NA27344.

${ }^{1} \mathrm{~S}$. Atzeni and J. Meyer ter Vehn, The Physics of Inertial Fusion (Oxford University Press, Oxford, U.K., 2007).

${ }^{2}$ G. H. Miller, E. I. Moses, and C. R. Wuest, Nuclear Fusion 44, S228 (2004).

${ }^{3}$ B. A. Hammel, Plasma Physics and Controlled Fusion 48, B497 (2006). 
${ }^{4}$ M. Herrmann, M. Tabak, and J. Lindl, Nuclear Fusion 41, 99 (2001).

${ }^{5}$ A. Kemp, J. Meyer-ter Vehn, and S. Atzeni, Phys. Rev. Lett. 86, 3336 (2001).

${ }^{6}$ O. L. Landen, T. R. Boehly, D. K. Bradley, D. G. Braun, D. A Callahan, P. M. Celliers, G. W. Collins, E. L. Dewald, L. Divol, S. H. Glenzer, et al., Physics of Plasmas 17, 056301 (2010).

${ }^{7}$ B. Spears, D. Hicks, C. Velsko, M. Stoyer, H. Robey, D. Munro, S. Haan, O. Landen, A. Nikroo, and H. Huang, Journal of Physics: Conference Series 112, 022003 (2008).

${ }^{8}$ B. A. Hammel, D. Griswold, O. L. Landen, T. S. Perry, B. A. Remington, P. L. Miller, T. A. Peyser, and J. D. Kilkenny, Physics of Fluids B: Plasma Physics 5, 2259 (1993).

${ }^{9}$ D. H. Kalantar, S. W. Haan, B. A. Hammel, C. J. Keane, O. L. Landen, and D. H. Munro, Rev. Sci. Instrum. 68, 814 (1997).

${ }^{10}$ F. J. Marshall, P. W. McKenty, J. A. Delettrez, R. Epstein, J. P. Knauer, V. A. Smalyuk, J. A. Frenje, C. K. Li, R. D. Petrasso, F. H. Séguin, et al., Phys. Rev. Lett. 102, 185004 (2009).

${ }^{11}$ The fuel velocity is very close to the ablator velocity during most of the accleration phase.

${ }^{12}$ Y. Saillard, Nuclear Fusion 46, 1017 (2006).

${ }^{13}$ The problem of a spatially-varying backlighter profile can be mitigated using point backlighters ${ }^{28}$ rather than area backlighters; however, these have far greater background levels in an indirectlydriven implosion experiment. Point backlighting does not mitigate spatial variations due to ablator blow-off.

${ }^{14}$ The NIF emulator capsule in this simulation is composed of 4 layers: A $23.1 \mu \mathrm{m}$ thick, $1.02 \mathrm{~g} / \mathrm{cm}^{3}$ inner layer of pure $\mathrm{CH}$; a $29.4 \mu \mathrm{m}$ thick, $1.07 \mathrm{~g} / \mathrm{cm}^{3}$ second layer composed of $\mathrm{CH}$ doped with 0.6 atomic percent of Germanium; a $12.8 \mu \mathrm{m}$ thick, 1.05 $\mathrm{g} / \mathrm{cm}^{3}$ third layer of $\mathrm{CH}$ doped with 0.4 atomic percent of Germanium; and a $68.3 \mu \mathrm{m}$ thick, $1.02 \mathrm{~g} / \mathrm{cm}^{3}$ outer pure $\mathrm{CH}$ layer. The 'pure CH' is actually composed of 42.4 atomic percent of carbon, 57.2 atomic percent of hydrogen, and 0.5 atomic percent of oxygen. The capsule is filled with a mixture of 7 atomic per- cent of deuterium and 97 atomic percent of ${ }^{3} \mathrm{He}$ gas at a total density of $8 \mathrm{mg} / \mathrm{cm}^{3}$.

${ }^{15}$ S. W. Haan, M. C. Herrmann, T. R. Dittrich, A. J. Fetterman, M. M. Marinak, D. H. Munro, S. M. Pollaine, J. D. Salmonson, G. L. Strobel, and L. J. Suter, Physics of Plasmas 12, 056316 (2005).

${ }^{16}$ C. J. Dasch, Appl. Opt. 31, 1146 (1992).

${ }^{17}$ W. H. Press, S. A. Teukolsky, W. T. Vetterling, and B. P. Flannery, Numerical Recipes: The Art of Scientific Computing, 3rd Edition (Cambridge University Press, New York, 2007).

${ }^{18}$ Note this approach takes the $\rho(r)$ profiles at various times to be independent. A more elegant approach would be to construct a $\rho(r, t) 2$-D field that is self-consistent with both the data and the mass continuity equation. This is a task for future development.

${ }^{19}$ J. R. Fienup, Appl. Opt. 21, 2758 (1982).

${ }^{20}$ J. Workman and G. A. Kyrala, Rev. Sci. Instrum. 72, 678 (2001).

${ }^{21}$ C. A. Iglesias, M. H. Chen, V. Sonnad, and B. G. Wilson, J. Quant. Spect. Rad. Trans. 81, 227 (2003).

${ }^{22}$ J. T. Larsen and S. M. Lane, Journal of Quantitative Spectroscopy and Radiative Transfer 51, 179 (1994).

${ }^{23}$ P. T. Boggs, R. H. Byrd, and R. B. Schnabel, SIAM J. Sci. Stat. Comput. 8, 1052 (1987).

${ }^{24}$ C. Sorce, J. Schein, F. Weber, K. Widmann, K. Campbell, E. Dewald, R. Turner, O. Landen, K. Jacoby, P. Torres, et al., Rev. Sci. Instrum. 77, 10E518 (2006).

${ }^{25}$ T. R. Boehly, D. L. Brown, R. S. Craxton, R. L. Keck, J. P. Knauer, J. H. Kelly, T. J. Kessler, S. A. Kumpan, S. J. Loucks, S. A. Letzring, et al., Optics Communications 133, 495 (1997).

${ }^{26}$ D. H. Kalantar, P. M. Bell, T. S. Perry, N. Sewall, J. Kimbrough, F. Weber, C. Diamond, and K. Piston, Rev. Sci. Instrum. 72, 751 (2001).

${ }^{27}$ G. B. Zimmerman and W. L. Kruer, Comments Plasma Phys. Controlled Fusion 2, 51 (1975).

${ }^{28}$ D. K. Bradley, O. L. Landen, A. B. Bullock, S. G. Glendinning, and R. E. Turner, Opt. Lett. 27, 134 (2002). 